\title{
Novel Penicillins Synthesized by Biotransformation Using Laccase from Trametes spec.
}

\author{
Annett Mikolasch, ${ }^{*}, a$ Timo Horst Johannes Niedermeyer,${ }^{b}$ Michael Lalk, ${ }^{b}$ Sabine Witt, ${ }^{c}$ \\ Simone Seefeldt,${ }^{c}$ Elke Hammer, ${ }^{a}$ Frieder Schauer, ${ }^{a}$ Manuela Gesell,${ }^{d}$ Susanne Hessel,${ }^{d}$ \\ Wolf-Dieter JÜLICH, ${ }^{b}$ and Ulrike LINDEQUIST ${ }^{b}$ \\ ${ }^{a}$ Institute of Microbiology, and ${ }^{b}$ Institute of Pharmacy, Ernst-Moritz-Arndt-University Greifswald; 17489 Greifswald, F.- \\ L.-Jahn-Str. 15, Germany: ${ }^{c}$ Ganomycin Society of Biomedical Research Ltd.; Greifswald, Germany: and ${ }^{d}$ Institute of \\ Marine Biotechnology; Germany.
}

Received November 13, 2005; accepted January 24, 2006

Eight novel penicillins were synthesized by heteromolecular reaction of ampicillin or amoxicillin with 2,5-dihydroxybenzoic acid derivatives using a laccase from Trametes spec. All products inhibited the growth of several gram positive bacterial strains in the agar diffusion assay, among them methicillin-resistant Staphylococcus aureus and vancomycin-resistant Enterococci. The products protected mice against an infection with Staphylococcus aureus lethal to the untreated animals. Cytotoxicity and acute toxicity of the new compounds were neglectable. The results show the usefulness of laccase for the synthesis of potential new antibiotics. The biological activity of the new compounds stimulates intensified pharmacological tests.

Key words penicillin; laccase; amoxicillin; ampicillin; biotransformation; resistance

There is an urgent need to develop new antimicrobial agents due to increasing bacterial resistance to therapeutically used drugs. Most methicillin-resistent Staphylococcus aureus (MRSA) strains are resistent not only to $\beta$-lactams, but also to most other antimicrobial agents. ${ }^{1)}$ Penicillin resistance among Streptococcus pneumoniae strains is widely accepted as a global problem. ${ }^{2-5)}$ Bacteria have developed several strategies for escaping the lethal action of $\beta$-lactams. It may be expected that specific circumstance will make one the more effective stragegy than the other. $\left.{ }^{6}\right)$ Much effort has been devoted to the discovery of drugs which would not be cleaved by $\beta$-lactamases of pathogenic strains and which have suitable physicochemical and pharmacodynamic profiles. ${ }^{7,8)}$ The modifications of $\beta$-lactam antibiotics could not keep pace with the development of resistance in the pathogenic microorganisms, so that numerous bacteria, among them multidrug resistant Staphylococcus strains, can no longer be treated with the currently available $\beta$-lactam antibiotics. $^{1,9,10)}$

Besides the modification of existing antibiotics by chemical or biochemical methods the coupling of presently used antibiotics with other bioactive compounds or components from them which are not in use till now is a promising way to generate novel molecules with improved therapeutic properties.

Laccase (benzenediol:oxygen oxidoreductase, EC 1.10.3.2), classically considered a hydroquinone oxidizing enzyme, is able to oligomerize molecules. Up to now main application fields of this enzyme are waste detoxification, textile dye transformation, biosensors and diagnostic application, where the capability to catalyze polymerization reactions is used. ${ }^{11-13)}$

Recently we reported about our synthetic results on coupling reactions with laccase. ${ }^{14-17)}$ Now we have employed laccase to achieve derivatisation of $\beta$-lactam antibiotics and to couple them with derivatives of 2,5-dihydroxybenzoic acid. These derivatives are structurally related to the ganomycins, a new chemical class of antibacterial com- pounds ${ }^{18)}$ and to other antibacterial active isolates ${ }^{19,20)}$ therefore interesting as coupling partner for $\beta$-lactams using laccase as initiator of the reaction to produce novel hybridantibiotics by biotransformation.

The use of laccase for the derivatisation of antibiotics is limited to a few examples including the phenolic oxidation of 7-(4-hydroxyphenylacetamido)cephalosporinic acid, ${ }^{21)}$ the dimerization of penicillin $\mathrm{X}^{22)}$ and the oxidative coupling of hydroquinone and mithramicine. ${ }^{23)}$ In the examples realized to date, the sought object of enhancement of the bioactive effect has not been achieved. ${ }^{21-23)}$

The aim of this study was (i) to investigate whether laccase can be used for the synthesis of novel penicillins by heteromolecular coupling of two different compounds, (ii) to characterize the products of the reaction, and (iii) to analyze the biological activity of the novel penicillins.

\section{Results and Discussion}

Biotransformation of Amoxicillin and Ampicillin by Laccase of Trametes spec. Laccase catalyzed reaction between amoxicillin or ampicillin (educts $\mathbf{2 a}, \mathbf{b}$ ) on one hand and a derivative of 2,5-dihydroxybenzoic acid (substrates 1a to d) on the other hand leads in any case to one cross coupling product (3a to h, Fig. 1).

High-performance liquid chromatography (HPLC) analysis of the reaction mixture revealed the simultaneous decrease of the two substrates and the increase of one product at each case. Sufficient amount of the products $\mathbf{3 a}$ to $\mathbf{h}$ (around 98\% yield) was formed over an incubation period of $3 \mathrm{~h}$. Comparable straight forward biotransformation of educts to products we found also for hybrid dimer formation from 2,5-dihydroxybenzoic acid derivatives with primary aromatic amines $^{14,17)}$ and from 3-(3,4-dihydroxy-phenyl)-propionic acid with 1-hexylamine. ${ }^{15)}$ These findings are in contrast to the reaction kinetics described for hybrid dimer formation from 3,4-dichloroaniline and syringic acid ${ }^{27)}$ or 3-(3,4-dihydroxyphenyl)-propionic acid and 4-aminobenzoic acid. ${ }^{15)}$ Therefore the laccase catalyzed amination of 2,5-dihydroxybenzoic 
<smiles>[R2]c1ccc(C(N)C(=O)NC2C(=O)N3C(C(=O)O)C(C)(C)S[C@H]23)cc1</smiles>

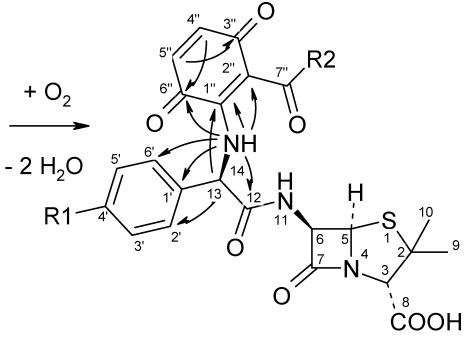

$1 a-1 d$

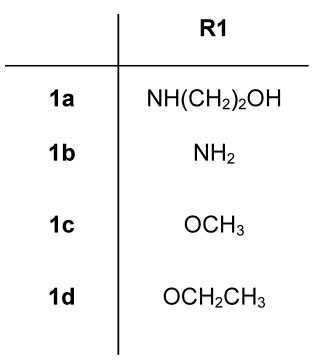

$2 a-2 b$

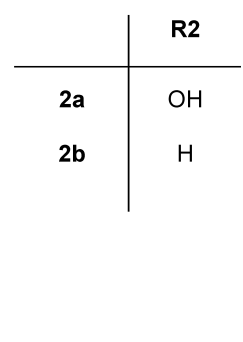

$3 a-3 h$

\begin{tabular}{|c|c|c|}
\hline & R1 & R2 \\
\hline $3 a$ & $\begin{array}{l}8^{\prime \prime} 9^{\prime \prime} 10^{\prime \prime} \\
\mathrm{NH}\left(\mathrm{CH}_{2}\right)_{2} \mathrm{OH}\end{array}$ & $\mathrm{OH}$ \\
\hline $3 b$ & $\begin{array}{c}8^{\prime \prime} \\
\mathrm{NH}_{2}\end{array}$ & $\mathrm{OH}$ \\
\hline $3 c$ & $\begin{array}{l}8^{\prime \prime} \\
\mathrm{OCH}_{3}\end{array}$ & $\mathrm{OH}$ \\
\hline $3 d$ & $\begin{array}{c}8 " 9^{\prime \prime} \\
\mathrm{OCH}_{2} \mathrm{CH}_{3}\end{array}$ & $\mathrm{OH}$ \\
\hline $3 e$ & $\begin{array}{l}8^{\prime \prime} 9^{\prime \prime} 10^{\prime \prime} \\
\mathrm{NH}\left(\mathrm{CH}_{2}\right)_{2} \mathrm{OH}\end{array}$ & $\mathrm{H}$ \\
\hline $3 f$ & $\begin{array}{l}8^{\prime \prime} \\
\mathrm{NH}_{2}\end{array}$ & $\mathrm{H}$ \\
\hline $3 \mathrm{~g}$ & $\begin{array}{c}8^{\prime \prime} \\
\mathrm{OCH}_{3}\end{array}$ & $\mathrm{H}$ \\
\hline $3 \mathrm{~h}$ & $\begin{array}{c}8 " 9 " \\
\mathrm{OCH}_{2} \mathrm{CH}_{3}\end{array}$ & $\mathrm{H}$ \\
\hline
\end{tabular}

Fig. 1. 2,5-Dihydroxybenzoic Acid Derivatives (1a to d), $\beta$-Lactam Antibiotics (2a, b) and the Products $\mathbf{3 a}$ to $\mathbf{h}$

Important HMBC correlations of compound 3a.

acid derivatives is an excellent method for the synthesis of novel penicillins.

After separation of the products $\mathbf{3} \mathbf{a}$ to $\mathbf{h}$ from buffer and laccase, mass spectral analyses (LC/MS with API-ES in both negative and positive modes) of the compounds showed a molecular mass attributed to the coupling of one 2,5-dihydroxybenzoic acid derivative (1a to $\mathbf{d}$ ) and one penicillin derivative ( $2 \mathbf{a}$ to $\mathbf{b}$ ). These couplings were confirmed by the presence of all carbons of the 2,5-dihydroxy- $N$-(2-hydroxyethyl)benzamide $\mathbf{1 a}$ and of amoxicillin $\mathbf{2} \mathbf{a}$ or ampicillin $\mathbf{2} \mathbf{b}$ in the ${ }^{13} \mathrm{C}-\mathrm{NMR}$ spectra of the products $\mathbf{3 a}$ and $\mathbf{e}$. Two signals in the range of $180 \mathrm{ppm}$ indicated a quinonoid character of the products. Furthermore, all proton signals of each of the two educts, beside the proton of the C-6 of the 2,5-dihydroxybenzoic acid derivatives, were detectable in the ${ }^{1} \mathrm{H}-\mathrm{NMR}$ spectra of the products. The number of $\mathrm{CH}$ proton signals of the dihydroxylated phenyl rings changed from three-in the educts-to two signals-in the products. The multiplicity of the signals of the C-4" and C-5" protons indicated a further substituent at the $\mathrm{C}-1^{\prime \prime}$ position. The chemical shift to lower field of the C-4" and C-5" protons demonstrated the presence of an electron-withdrawing group. The HMBC spectrum of $\mathbf{3 a}$ (Table 1, Fig. 1) showed correlations between the aliphatic amine proton $\mathrm{H}-14$ and the $\mathrm{C}-6^{\prime \prime}$ quinone carbonylcarbon and the $\mathrm{C}-1^{\prime \prime}$ and $\mathrm{C}-2^{\prime \prime}$ carbons of the quinone ring. A correlation in the $\mathrm{HMBC}$ could be seen between the aliphatic $\mathrm{CH}$ proton $\mathrm{H}-13$ of the amoxicillin and the $\mathrm{C}-1^{\prime \prime}$ carbon of the quinone ring. These facts proofed $\mathbf{3} \mathbf{a}$ to be aminated at the $\mathrm{C}-1$ " position of the quinone ring. The HMBC included also correlation between the $\mathrm{C}-4$ " and $\mathrm{C}-5^{\prime \prime}$ protons of the quinone ring
Table 1. ${ }^{1} \mathrm{H}$ and ${ }^{13} \mathrm{C}$ Assignments and $\mathrm{HMBC}$ Correlations for 3a

\begin{tabular}{|c|c|c|c|}
\hline Carbon & ${ }^{13} \mathrm{C}^{a)}$ & ${ }^{1} \mathrm{H}^{b)}$ & ${ }^{1} \mathrm{H}-{ }^{13} \mathrm{C}$ correlations \\
\hline 9,10 & $27.06,31.62$ & $1.43, \mathrm{~s}, 3 \mathrm{H}, 1.52, \mathrm{~s}, 3 \mathrm{H}$ & $\mathrm{C} 2, \mathrm{C} 3$ \\
\hline $9^{\prime \prime}$ & 42.10 & $3.37, \mathrm{~m}, 2 \mathrm{H}(5.5)$ & $\mathrm{C}^{\prime}$ \\
\hline 6 & 59.61 & $5.52, \mathrm{dd}, 1 \mathrm{H}(4.2)$ & $\mathrm{C} 7, \mathrm{C} 12$ \\
\hline 13 & 62.77 & $5.92, \mathrm{~d}, 1 \mathrm{H}(6.6)$ & $\mathrm{C} 12, \mathrm{C} 2^{\prime}, \mathrm{C} 6^{\prime}, \mathrm{C} 1^{\prime \prime}$ \\
\hline 5 & 68.36 & $5.38, \mathrm{~d}, 1 \mathrm{H}(4.2)$ & C7 \\
\hline 3 & 71.15 & $4.30, \mathrm{~s}, 1 \mathrm{H}$ & $\mathrm{C} 2, \mathrm{C} 5, \mathrm{C} 7, \mathrm{C} 8, \mathrm{C} 9, \mathrm{C} 10$ \\
\hline $3^{\prime}, 5^{\prime}$ & 116.63 & $6.81, \mathrm{~d}, 2 \mathrm{H}(8.6)$ & $\begin{array}{l}\mathrm{C} 1^{\prime}, \mathrm{C} 2^{\prime}, \mathrm{C} 3^{\prime}, \mathrm{C} 4^{\prime}, \mathrm{C} 5^{\prime}, \\
\mathrm{C} 6^{\prime}\end{array}$ \\
\hline $2^{\prime}, 6^{\prime}$ & 129.95 & $7.27, \mathrm{~d}, 2 \mathrm{H}(8.6)$ & $\mathrm{C} 2^{\prime}, \mathrm{C} 3^{\prime}, \mathrm{C} 4^{\prime}, \mathrm{C} 5^{\prime}, \mathrm{C} 6^{\prime}$ \\
\hline 4" & 133.32 & $6.55, \mathrm{~d}, 1 \mathrm{H}(10.2)$ & $\mathrm{C} 1 ", \mathrm{C} 2 ", \mathrm{C} 6^{\prime \prime}, \mathrm{C} 7 "$ \\
\hline $5^{\prime \prime}$ & 141.93 & $6.66, \mathrm{~d}, 1 \mathrm{H}(10.2)$ & $\mathrm{C} 3^{\prime \prime}, \mathrm{C} 1^{\prime \prime}$ \\
\hline \multicolumn{2}{|c|}{ NH (11) } & $7.27, \mathrm{~d}, 1 \mathrm{H}$ & C5, C6, C7, C12, C13 \\
\hline \multicolumn{2}{|l|}{ NH (14) } & $13.13, \mathrm{~d}, 1 \mathrm{H}(6.2)$ & $\begin{array}{l}\mathrm{C} 12, \mathrm{C} 1^{\prime}, \mathrm{C} 2^{\prime}, \mathrm{C} 6^{\prime}, \mathrm{C} 1^{\prime \prime}, \\
\mathrm{C} 2^{\prime \prime}, \mathrm{C} 6^{\prime \prime}\end{array}$ \\
\hline $\mathrm{NH}\left(8^{\prime \prime}\right)$ & & $9.77, \mathrm{t}, 1 \mathrm{H}$ & $\mathrm{C} 9^{\prime \prime}$ \\
\hline
\end{tabular}

a) Chemical shifts are expressed in \% (ppm) calibrated on the resonances of the residual nondeuterated solvent. b) ${ }^{1} \mathrm{H}, J(\mathrm{in} \mathrm{Hz})$ values in parentheses. Determination of proton assignment by HSQC.

and the quinone carbonyl carbons C-3" and C- 6 ", confirming the oxidation of the $p$-hydroquinone to a quinone as described previously for hybrid dimer formation from 2,5-dihydroxybenzoic acid derivatives with primary aromatic amines, ${ }^{14,17)}$ and for the hybrid dimer formation from 3,4-dichloraniline with protocatechuic acid and syringic acid. ${ }^{28,27)}$

Biological Activity of the Biotransformation Products All products obtained by biotransformation according to Fig. 1 ( $3 \mathbf{a}$ to $\mathbf{h})$ showed a moderate to strong growth inhibition of several gram positive strains, among them multidrug resist- 
Table 2a. Antimicrobial Activity of Products $\mathbf{3 a}$ to $\mathbf{d}$ and $\mathbf{2 a}$

\begin{tabular}{|c|c|c|c|c|c|c|c|c|c|c|c|c|c|c|c|}
\hline \multirow{3}{*}{ Strain } & \multicolumn{15}{|c|}{ Amount $n[\mu \mathrm{mol}]$} \\
\hline & \multicolumn{3}{|c|}{$2 \mathbf{a}$} & \multicolumn{3}{|c|}{$3 \mathbf{a}$} & \multicolumn{3}{|c|}{$3 \mathbf{b}$} & \multicolumn{3}{|c|}{$3 c$} & \multicolumn{3}{|c|}{ 3d } \\
\hline & 0.019 & 0.1 & 0.19 & 0.019 & 0.1 & 0.19 & 0.019 & 0.1 & 0.19 & 0.019 & 0.1 & 0.19 & 0.029 & 0.14 & 0.29 \\
\hline $\begin{array}{l}\text { Bacillus megaterium } \\
\text { SBUG } 1152\end{array}$ & $26^{a)}$ & 30 & 34 & 20 & 24 & 24 & 20 & 24 & 24 & 20 & 24 & 24 & 18 & 22 & 24 \\
\hline B. subtils AWD 166 & 30 & 36 & 38 & 24 & 30 & 30 & 24 & 30 & 30 & 24 & 30 & 30 & 20 & 28 & 30 \\
\hline $\begin{array}{l}\text { Escherichia coli } \\
\text { SBUG } 1135\end{array}$ & 18 & 24 & 28 & 16 & 20 & 24 & 10 & 18 & 22 & 16 & 20 & 24 & $\mathrm{r}^{b)}$ & 18 & 20 \\
\hline $\begin{array}{l}\text { Enterococcus faecalis } \\
769\end{array}$ & 24 & 30 & 34 & 14 & 20 & 24 & 18 & 22 & 24 & 14 & 20 & 24 & 18 & 20 & 22 \\
\hline E. faecalis 945 & 20 & 26 & 28 & 10 & 20 & 24 & 10 & 18 & 20 & 10 & 20 & 24 & $\mathrm{r}$ & 18 & 20 \\
\hline $\begin{array}{l}\text { Staphylococcus aureus } \\
315\end{array}$ & 8 & 14 & 16 & $\mathrm{r}$ & 8 & 12 & $\mathrm{r}$ & 10 & 14 & $\mathrm{r}$ & 8 & 12 & $\mathrm{r}$ & 8 & 10 \\
\hline S. aureus 33490 & 18 & 22 & 24 & 14 & 20 & 24 & 12 & 18 & 20 & 14 & 20 & 24 & $\mathrm{r}$ & 14 & 18 \\
\hline S. aureus 34289 & $\mathrm{r}$ & 10 & 12 & $\mathrm{r}$ & $\mathrm{r}$ & 8 & $\mathrm{r}$ & 8 & 12 & $\mathrm{r}$ & $\mathrm{r}$ & 8 & $\mathrm{r}$ & $\mathrm{r}$ & 8 \\
\hline S. aureus 36881 & 8 & 14 & 16 & 8 & 12 & 16 & $\mathrm{r}$ & 12 & 16 & 8 & 12 & 16 & $\mathrm{r}$ & 10 & 12 \\
\hline S. aureus 38418 & 34 & 40 & 42 & 24 & 30 & 36 & 28 & 30 & 34 & 24 & 30 & 36 & 24 & 30 & 32 \\
\hline S. aureus 39105 & 12 & 16 & 18 & $\mathrm{r}$ & 10 & 14 & $\mathrm{r}$ & 10 & 16 & $\mathrm{r}$ & 10 & 14 & $\mathrm{r}$ & 10 & 12 \\
\hline S. aureus 520 & $\mathrm{r}$ & 14 & 16 & $\mathrm{r}$ & 8 & 10 & $\mathrm{r}$ & 8 & 12 & $\mathrm{r}$ & 8 & 10 & $\mathrm{r}$ & 8 & 10 \\
\hline S. aureus 526 & $\mathrm{r}$ & 12 & 16 & $\mathrm{r}$ & 10 & 12 & $\mathrm{r}$ & $\mathrm{r}$ & 10 & $\mathrm{r}$ & 10 & 12 & $\mathrm{r}$ & $\mathrm{r}$ & $\mathrm{r}$ \\
\hline S. aureus ATCC 6538 & 36 & 42 & 44 & 26 & 30 & 34 & 28 & 32 & 36 & 26 & 30 & 34 & 30 & 32 & 34 \\
\hline $\begin{array}{l}\text { S. aureus Nordeutscher } \\
\text { Stamm }\end{array}$ & $\mathrm{r}$ & 12 & 14 & $\mathrm{r}$ & 10 & 14 & $\mathrm{r}$ & 12 & 16 & $\mathrm{r}$ & 10 & 14 & $\mathrm{r}$ & 8 & 10 \\
\hline S. epidermidis 1068 & 20 & 24 & 26 & 14 & 20 & 22 & 16 & 20 & 22 & 14 & 20 & 22 & 16 & 18 & 20 \\
\hline S. epidermidis 1071 & 24 & 26 & 28 & 14 & 20 & 22 & 14 & 20 & 22 & 14 & 20 & 22 & 12 & 18 & 20 \\
\hline S. epidermidis 125 & 30 & 36 & 38 & 14 & 20 & 24 & 20 & 26 & 28 & 14 & 20 & 24 & 22 & 24 & 26 \\
\hline S. epidermidis 535 & $\mathrm{r}$ & 8 & 12 & $\mathrm{r}$ & $\mathrm{r}$ & $\mathrm{r}$ & $\mathrm{r}$ & $\mathrm{r}$ & 8 & $\mathrm{r}$ & $\mathrm{r}$ & $\mathrm{r}$ & $\mathrm{r}$ & $\mathrm{r}$ & 8 \\
\hline S. epidermidis 563 & 10 & 16 & 18 & 8 & 16 & 18 & 8 & 14 & 18 & 8 & 16 & 18 & $\mathrm{r}$ & 14 & 18 \\
\hline S. epidermidis 847 & 20 & 20 & 28 & $\mathrm{r}$ & 16 & 20 & 28 & 30 & 32 & $\mathrm{r}$ & 16 & 20 & 8 & 14 & 18 \\
\hline
\end{tabular}

a) Zones of inhibition (mm). b) $\mathrm{r}$ resistent (no zone of inhibition).

Table 2b. Antimicrobial Activity of Products $\mathbf{3 e}$ to $\mathbf{h}$ and $\mathbf{2 b}$

\begin{tabular}{|c|c|c|c|c|c|c|c|c|c|c|c|c|c|c|c|}
\hline \multirow{3}{*}{ Strain } & \multicolumn{15}{|c|}{ Amount $n[\mu \mathrm{mol}]$} \\
\hline & \multicolumn{3}{|c|}{$2 \mathbf{b}$} & \multicolumn{3}{|c|}{$3 e$} & \multicolumn{3}{|c|}{$3 f$} & \multicolumn{3}{|c|}{$3 g$} & \multicolumn{3}{|c|}{$3 \mathbf{h}$} \\
\hline & 0.019 & 0.1 & 0.19 & 0.029 & 0.14 & 0.29 & 0.02 & 0.1 & 0.2 & 0.029 & 0.14 & 0.29 & 0.019 & 0.1 & 0.19 \\
\hline $\begin{array}{l}\text { Bacillus megaterium } \\
\text { SBUG } 1152\end{array}$ & $28^{a)}$ & 32 & 34 & 18 & 22 & 26 & 20 & 28 & 30 & 24 & 28 & 30 & 20 & 22 & 24 \\
\hline B. subtils AWD 166 & 32 & 34 & 36 & 24 & 30 & 30 & 28 & 30 & 32 & 26 & 30 & 32 & 22 & 28 & 30 \\
\hline $\begin{array}{l}\text { Escherichia coli } \\
\text { SBUG } 1135\end{array}$ & 24 & 30 & 34 & 10 & 20 & 24 & 16 & 28 & 30 & 22 & 30 & $>30$ & 14 & 20 & 28 \\
\hline $\begin{array}{l}\text { Enterococcus faecalis } \\
769\end{array}$ & 22 & 28 & 30 & 18 & 26 & 28 & 18 & 24 & 28 & 22 & 28 & 30 & 14 & 20 & 24 \\
\hline E. faecalis 945 & 18 & 28 & 30 & $\mathrm{r}^{b)}$ & 20 & 22 & 10 & 20 & 22 & 14 & 22 & 24 & $\mathrm{r}$ & 8 & 10 \\
\hline $\begin{array}{l}\text { Staphylococcus aureus } \\
315\end{array}$ & 10 & 16 & 18 & $\mathrm{r}$ & 8 & 12 & $\mathrm{r}$ & 12 & 14 & $\mathrm{r}$ & 12 & 14 & $\mathrm{r}$ & 10 & 12 \\
\hline S. aureus 33490 & 22 & 26 & 30 & 14 & 20 & 22 & 16 & 20 & 24 & 20 & 24 & 26 & 14 & 18 & 20 \\
\hline S. aureus 34289 & $\mathrm{r}$ & 12 & 16 & $\mathrm{r}$ & $\mathrm{r}$ & 10 & $\mathrm{r}$ & 14 & 18 & $\mathrm{r}$ & 14 & 18 & $\mathrm{r}$ & $\mathrm{r}$ & 12 \\
\hline S. aureus 36881 & 10 & 16 & 20 & $\mathrm{r}$ & 14 & 16 & 8 & 14 & 18 & 12 & 18 & 22 & $\mathrm{r}$ & 12 & 16 \\
\hline S. aureus 38418 & 38 & 42 & 44 & 28 & $>30$ & $>30$ & 32 & 36 & 40 & 28 & $>30$ & $>30$ & 28 & 32 & 36 \\
\hline S. aureus 39105 & 14 & 18 & 20 & $r$ & 12 & 14 & $\mathrm{r}$ & 14 & 16 & 10 & 16 & 18 & $\mathrm{r}$ & 12 & 14 \\
\hline S. aureus 520 & 8 & 14 & 16 & $\mathrm{r}$ & $\mathrm{r}$ & 12 & $\mathrm{r}$ & 14 & 18 & $\mathrm{r}$ & 10 & 12 & $\mathrm{r}$ & 10 & 14 \\
\hline S. aureus 526 & $\mathrm{r}$ & 14 & 16 & $\mathrm{r}$ & $\mathrm{r}$ & 10 & $\mathrm{r}$ & 10 & 12 & $r$ & 12 & 14 & $\mathrm{r}$ & $r$ & 12 \\
\hline S. aureus ATCC 6538 & 40 & 44 & 48 & $>30$ & $>30$ & $>30$ & 30 & 34 & 38 & 14 & 20 & 24 & 30 & 32 & 36 \\
\hline $\begin{array}{l}\text { S. aureus Nordeutscher } \\
\text { Stamm }\end{array}$ & r 10 & 14 & 18 & r & 8 & 12 & $\mathrm{r}$ & 14 & 16 & 8 & 16 & 18 & $\mathrm{r}$ & 12 & 14 \\
\hline S. epidermidis 1068 & 22 & 26 & 30 & 18 & 22 & 24 & 18 & 20 & 22 & 18 & 22 & 24 & 16 & 18 & 20 \\
\hline S. epidermidis 1071 & 26 & 30 & 32 & 16 & 18 & 20 & 18 & 24 & 28 & 18 & 26 & 28 & 16 & 20 & 22 \\
\hline S. epidermidis 125 & 34 & 38 & 40 & 18 & 20 & 22 & 26 & 30 & 32 & 20 & 26 & 28 & 20 & 26 & 28 \\
\hline S. epidermidis 535 & $\mathrm{r}$ & 10 & 12 & $\mathrm{r}$ & $\mathrm{r}$ & 8 & $\mathrm{r}$ & 8 & 12 & $\mathrm{r}$ & $\mathrm{r}$ & 10 & $\mathrm{r}$ & $\mathrm{r}$ & $\mathrm{r}$ \\
\hline S. epidermidis 563 & 20 & 22 & 28 & $\mathrm{r}$ & 18 & 20 & 10 & 18 & 20 & 16 & 24 & 26 & $\mathrm{r}$ & 16 & 18 \\
\hline S. epidermidis 847 & 26 & 28 & 30 & 14 & 20 & 22 & 16 & 22 & 26 & 16 & 24 & 26 & 10 & 20 & 22 \\
\hline
\end{tabular}

a) Zones of inhibition (mm). b) $\mathrm{r}$ resistent (no zone of inhibition). 
ant Staphylococcus and Enterococcus strains, and of the gram negative strain $E$. coli in the agar diffusion assay (Tables $2 \mathrm{a}, \mathrm{b}$ ), but not against Pseudomonas aeruginosa and $P$. maltophilia. Investigations towards the stability of the synthesised compounds showed limited lifetime in aqueous solution. Incubation of solutions of compounds $\mathbf{3 a}$ to $\mathbf{h}$ at $30^{\circ} \mathrm{C}$ showed decomposition after $2 \mathrm{~h}$. Therefore the survey of the antimicrobial effects were concentrated on the initial screening using the agar diffusion test. Because of no or only weak cytotoxicity of $\mathbf{3 a}$ to $\mathbf{h}$ against FL cells in concentrations up to $100 \mu \mathrm{g} / \mathrm{ml}$ (data not shown), a "Staphylococcus-infected, immune suppressed mouse" model was used for the examination of in vivo effectiveness of in vitro active products $3 \mathbf{a}$ to $\mathbf{h}$ (with exception of $\mathbf{3 g}$, which was not tested, Table 3 ).

All mice treated i.p. with one of the biotransformation products survived the infection with Staphylococcus aureus ATCC 6538 or the multidrug resistant strain Staphylococcus aureus 38418 whereas all untreated mice died after infection within $2 \mathrm{~d}$. The treated mice did not show any signs of intoxication. Besides this, 3a was tested in higher concentration ( $2500 \mathrm{mg} / \mathrm{kg}$ i.p., observation period $20 \mathrm{~d}$ after injection) or repeated application $(50 \mathrm{mg} / \mathrm{kg}$ i.p. two times per day for $14 \mathrm{~d}$, observation period $30 \mathrm{~d}$ starting with the first day of injection) for possible toxic effects in mice. Both schedules did not show any toxic side effects during the observation period.
These results showed that derivatization of antibiotics by laccase catalyzed reaction can be done without a loss of antibacterial activity. Several attempts have been done to produce highly active antibiotics by the derivatization of bioactive compounds by laccase in other laboratories, as well. Most of them led to a distinct loss of antibacterial activity. ${ }^{21,22)}$

Unfortunately, the new derivatives produced within this study did not show a high increase in activity. No considerable differences between the eight coupling products or between coupling products and amoxicillin or ampicillin concerning spectrum and strength of antibacterial activity were found. However, derivatization can also influence other features of the antibacterial compounds favourable. Thus, coupling product 3a was quite stable against both tested types of $\beta$-lactamase whereas amoxicillin and ampicillin were more reduced in their activity against Staphylococcus aureus SG10 after treatment with $\beta$-lactamases (Table 4). Furthermore, the positive $\log \mathrm{D}$ values of the products $(\mathbf{3} \mathbf{a} \log \mathrm{D} 1.15, \mathbf{3 b} \log \mathrm{D}$ $1.64,3 \mathrm{c} \log \mathrm{D} 1.76,3 \mathrm{~d} \log \mathrm{D} 2.06,3 \mathrm{e} \log \mathrm{D} 2.12,3 \mathbf{f} \log \mathrm{D}$ $2.56, \mathbf{3 g} \log \mathrm{D} 2.72,3 \mathbf{h} \log \mathrm{D} 2.87$ ) in contrast to the negative $\log \mathrm{D}$ of the known antibiotics amoxicillin $(\mathbf{2} \mathbf{a} \log \mathrm{D}-2.84)$ and ampicillin $(\mathbf{2} \mathbf{b} \log \mathrm{D}-2.81)$ suggest that the compounds could be adsorbed from the gastrointestinal tract after peroral application. Their stability against acids is not clear in the moment.

Table 3. Effectiveness of in Vitro Active Products 3a to $\mathbf{h}$ in the "Staphylococcus-Infected, Immune Suppressed Mouse" Model

\begin{tabular}{|c|c|c|c|c|c|c|}
\hline \multirow{2}{*}{ Product } & Concentration & $\begin{array}{c}\text { Survived/treated } \\
\text { mice } n / n\end{array}$ & $\begin{array}{l}\text { Survived/control } \\
\text { mice } n / n\end{array}$ & Concentration & $\begin{array}{l}\text { Survived } / \text { treated } \\
\text { mice } n / n\end{array}$ & $\begin{array}{l}\text { Survived/control } \\
\text { mice } n / n\end{array}$ \\
\hline & \multicolumn{3}{|c|}{ Staphylococcus aureus ATCC 6538} & \multicolumn{3}{|c|}{ Staphylococcus aureus 38418} \\
\hline $\mathbf{2 a}$ & $\begin{array}{l}2 \times 0.5 \mathrm{mg} \\
(25 \mathrm{mg} / \mathrm{kg})\end{array}$ & $10 / 10$ & $0 / 10$ & $\begin{array}{l}3 \times 2.7 \mathrm{mg} 1 \mathrm{~d}^{a)} \\
2 \times 1.3 \mathrm{mg} 2 \mathrm{~d} \\
2 \times 1.3 \mathrm{mg} 3 \mathrm{~d} \\
2 \times 1.3 \mathrm{mg} 4 \mathrm{~d} \\
1 \times 1.3 \mathrm{mg} 5 \mathrm{~d} \\
1 \times 1.3 \mathrm{mg} 6 \mathrm{~d}\end{array}$ & $3 / 3$ & $0 / 5$ \\
\hline $2 \mathbf{b}$ & $\begin{array}{l}2 \times 0.5 \mathrm{mg} \\
(25 \mathrm{mg} / \mathrm{kg})\end{array}$ & $10 / 10$ & $0 / 10$ & $\begin{array}{l}3 \times 2.7 \mathrm{mg} 1 \mathrm{~d} \\
2 \times 1.3 \mathrm{mg} 2 \mathrm{~d} \\
2 \times 1.3 \mathrm{mg} 3 \mathrm{~d} \\
2 \times 1.3 \mathrm{mg} 4 \mathrm{~d} \\
1 \times 1.3 \mathrm{mg} 5 \mathrm{~d}\end{array}$ & $3 / 3$ & $1 / 5$ \\
\hline $3 \mathbf{a}$ & $\begin{array}{l}2 \times 0.5 \mathrm{mg} \\
(25 \mathrm{mg} / \mathrm{kg})\end{array}$ & $20 / 20$ & $0 / 20$ & $\begin{array}{l}3 \times 4 \mathrm{mg} 1 \mathrm{~d} \\
2 \times 2 \mathrm{mg} 2 \mathrm{~d} \\
2 \times 2 \mathrm{mg} 3 \mathrm{~d} \\
2 \times 2 \mathrm{mg} 4 \mathrm{~d}\end{array}$ & $3 / 3$ & $0 / 5$ \\
\hline $\mathbf{3 b}$ & $\begin{array}{l}2 \times 0.5 \mathrm{mg} \\
(25 \mathrm{mg} / \mathrm{kg})\end{array}$ & $6 / 6$ & $0 / 10$ & $\begin{array}{l}3 \times 4 \mathrm{mg} 1 \mathrm{~d} \\
2 \times 2 \mathrm{mg} 2 \mathrm{~d} \\
2 \times 2 \mathrm{mg} 3 \mathrm{~d} \\
2 \times 2 \mathrm{mg} 4 \mathrm{~d}\end{array}$ & $3 / 3$ & $0 / 5$ \\
\hline $3 c$ & $\begin{array}{l}2 \times 1.0 \mathrm{mg} \\
(50 \mathrm{mg} / \mathrm{kg})\end{array}$ & $3 / 3$ & $0 / 5$ & $-b)$ & - & - \\
\hline $3 d$ & $\begin{array}{l}2 \times 0.5 \mathrm{mg} \\
(25 \mathrm{mg} / \mathrm{kg})\end{array}$ & $6 / 6$ & $0 / 10$ & $\begin{array}{l}3 \times 4 \mathrm{mg} 1 \mathrm{~d} \\
2 \times 2 \mathrm{mg} 2 \mathrm{~d} \\
2 \times 2 \mathrm{mg} 3 \mathrm{~d} \\
2 \times 2 \mathrm{mg} 4 \mathrm{~d} \\
1 \times 2 \mathrm{mg} 5 \mathrm{~d} \\
1 \times 2 \mathrm{mg} 6 \mathrm{~d}\end{array}$ & $3 / 3$ & $0 / 5$ \\
\hline $3 e$ & $\begin{array}{l}2 \times 0.5 \mathrm{mg} \\
(25 \mathrm{mg} / \mathrm{kg})\end{array}$ & $9 / 9$ & $0 / 15$ & - & - & - \\
\hline $3 f$ & $\begin{array}{l}2 \times 2.0 \mathrm{mg} \\
(100 \mathrm{mg} / \mathrm{kg})\end{array}$ & $3 / 3$ & $0 / 5$ & - & - & - \\
\hline $3 h$ & $\begin{array}{l}2 \times 1.0 \mathrm{mg} \\
(50 \mathrm{mg} / \mathrm{kg})\end{array}$ & $3 / 3$ & $0 / 5$ & - & - & - \\
\hline
\end{tabular}


Table 4. Stability of $\beta$-Lactam Antibiotics and Product 3a against Two Types of $\beta$-Lactamase

\begin{tabular}{|c|c|c|c|}
\hline Substance & Control & $\begin{array}{l}\text { Inhibition } \\
\text { zone after } \\
\text { treatment } \\
\text { with } \beta \text { - } \\
\text { lactamase } 1\end{array}$ & $\begin{array}{c}\text { Inhibition } \\
\text { zone after } \\
\text { treatment } \\
\text { with } \beta \text { - } \\
\text { lactamase } 2\end{array}$ \\
\hline Penicillin G & $5.5 \mathrm{~cm}$ & $4.2 \mathrm{~cm}$ & —a) $^{a}$ \\
\hline Ampicillin & $5.2 \mathrm{~cm}$ & $4.1 \mathrm{~cm}$ & $3.7 \mathrm{~cm}$ \\
\hline Amoxicillin & $4.9 \mathrm{~cm}$ & $4.6 \mathrm{~cm}$ & $3.3 \mathrm{~cm}$ \\
\hline $3 \mathbf{a}$ & $4.0 \mathrm{~cm}$ & $3.9 \mathrm{~cm}$ & $3.4 \mathrm{~cm}$ \\
\hline
\end{tabular}

a) -, no inhibition zone after treatment with $\beta$-lactamase.

In total, the novel penicillins produced by biotransformation using laccase broaden the spectrum of $\beta$-lactam antibiotics. The transfer of the laccase catalyzed synthesis on other substrates, for example cephalosporins, shows the enormous potential of this method for the synthesis of new bioactive compounds. Further studies regarding the mode of action of the novel penicillins, acute and chronic toxicity, activity after peroral application and pharmacokinetic behaviour as well as investigations towards the enhancement of stability of these derivatives are in progress.

\section{Experimental}

Substrates and Conditions of Biotransformation Amoxicillin or ampicillin $(2 \mathrm{~mm}$ ) were dissolved in $600 \mathrm{ml}$ sodium acetate buffer, $20 \mathrm{~mm} \mathrm{pH}$ 5.6. After addition of laccase C (activity $800 \mathrm{nmol} \cdot \mathrm{ml}^{-1} \cdot \mathrm{min}^{-1} \cdot$ ) of Trametes spec. (EC 1.10.3.2, ASA Spezialenzyme GmbH Wolfenbüttel, Germany), the reaction mixture was completed by a $20 \mathrm{~mm}$ solution of one of the following 2,5-dihydroxybenzoic acid derivatives in $60 \mathrm{ml}$ sodium acetate buffer, pH 5.6: 2,5-dihydroxy- $N$-(2-hydroxyethyl)benzamide, 2,5-dihydroxybenzamide, 2,5-dihydroxybenzoic acid methyl ester or 2,5-dihydroxybenzoic acid ethyl ester. The reaction mixture was incubated for $3 \mathrm{~h}$ at room temperature (RT) with agitation at $400 \mathrm{rpm}$.

Chemicals were purchased from commercial suppliers: amoxicillin from Sigma-Aldich, ampicillin from AppliChem Darmstadt Germany, 2,5-dihydroxy- $N$-(2-hydroxyethyl)benzamide from Midori Kagaku Co Japan, 2,5-dihydroxybenzoic acid methyl ester and 2,5-dihydroxybenzoic acid ethyl ester from Sigma-Aldrich. All chemicals were used as received. 2,5-Dihydroxybenzamide was synthesized as described previously. ${ }^{17)}$

Analytical High-Performance Liquid Chromatography (HPLC) For routine analysis, samples of the incubation mixture were analyzed by a HPLC system (Dionex, Idstein, Germany) consisting of a Gynkotek High Precision Pump Model 480, Dionex ASI-100 Automated Sample-Injector, Dionex UVD 340S Diode-Array-Detector, and Chromeleon Version 6.30. An endcapped, 5- $\mu \mathrm{m}$, LiChroCart 125-4 RP 18 column (Merck, Darmstadt, Germany) was used. A solvent system consisting of methanol (eluent A) and phosphoric acid, $0.1 \% \mathrm{pH} 2$ (eluent $\mathrm{B}$ ), starting from an initial ratio of $10 \%$ $\mathrm{A}$ and $90 \% \mathrm{~B}$ and reaching $100 \% \mathrm{~A}$ within $14 \mathrm{~min}$, was used at a flow rate of $1 \mathrm{ml} / \mathrm{min}$

General Procedure for Isolation of Biotransformation Products After $3 \mathrm{~h}$ the two educts of each reaction were transformed by laccase in any case to one product. After activation and equilibration, a RP18 silicagel column ( $20 \mathrm{cc}, 5 \mathrm{~g}$ absorbent material, Waters, Ireland) was charged with $200 \mathrm{ml}$ of the incubation mixture. The column was washed twice with $20 \mathrm{ml}$ of a mixture of methanol $(10 \%)$ and destilled water $(90 \%)$. The products were eluted with $10 \mathrm{ml}$ acetonitrile. This routine procedure was repeated four times for each reaction. The combined acetonitrile eluates were dried using a vacuum rotator at $30^{\circ} \mathrm{C}$.

Characterization of Biotransformation Products Products were analyzed by mass spectrometry (LC/MS with API-ES in both negative and positive modes).

The nuclear magnetic resonance (NMR) spectra were obtained at $300 \mathrm{MHz}\left({ }^{1} \mathrm{H}\right)$, at $75 \mathrm{MHz}\left({ }^{13} \mathrm{C}\right.$ and DEPT-135), and at $600 \mathrm{MHz}$ (HSQC, $\mathrm{HMBC})$ in acetonitrile- $d_{3}$.

6-\{2-[2-(2-Hydroxyethylcarbamoyl)-3,6-dioxocyclohexa-1,4-dienyl- amino]-2-(4-hydroxyphenyl)-acetylamino\}-penicillanic Acid 3a Synthesis and isolation as described above. Dark red solid. ${ }^{1} \mathrm{H}-\mathrm{NMR} \delta 1.43$ (s, 3H, H-9 or H-10), 1.52 (s, 3H, H-9 or H-10), 3.37 (m, J=5.5 Hz, 2H, H$\left.9^{\prime \prime}\right), 3.57$ (t, $\left.J=5.5 \mathrm{~Hz}, 2 \mathrm{H}, \mathrm{H}-10^{\prime \prime}\right), 4.30$ (s, $1 \mathrm{H}, \mathrm{H}-3$ ), 5.38 (d, $J=4.2 \mathrm{~Hz}, 1 \mathrm{H}$, H-5), 5.52 (dd, $J=4.2 \mathrm{~Hz}, 1 \mathrm{H}, \mathrm{H}-6), 5.92$ (d, $J=6.6 \mathrm{~Hz}, 1 \mathrm{H}, \mathrm{H}-13$ ), 6.55 $\left(\mathrm{d}, J=10.2 \mathrm{~Hz}, 1 \mathrm{H}, \mathrm{H}-4^{\prime \prime}\right), 6.66\left(\mathrm{~d}, J=10.2 \mathrm{~Hz}, 1 \mathrm{H}, \mathrm{H}-5^{\prime \prime}\right), 6.81$ (d, $J=8.6 \mathrm{~Hz}$, 2H, H-3', H-5'), 7.27 (d, $J=8.6 \mathrm{~Hz}, 2 \mathrm{H}, \mathrm{H}-2^{\prime}, \mathrm{H}-6^{\prime}$ ), 7.27 (d, 1H, H-11), $9.77\left(\mathrm{t}, 1 \mathrm{H}, \mathrm{H}-8^{\prime \prime}\right), 13.13$ (d, $\left.1 \mathrm{H}, J=6.2 \mathrm{~Hz}, \mathrm{H}-14\right) .{ }^{13} \mathrm{C}-\mathrm{NMR} \delta 27.06$ (C-9 or C-10), 31.62 (C-9 or C-10), 42.10 (C-9"), 59.61 (C-6), 61.52 (C-10"), 62.77 (C-13), 64.90 (C-2), 68.36 (C-5), 71.15 (C-3), 101.42 (C-1"), 116.63 (C-3', $\left.\mathrm{C}-5^{\prime}\right), 129.82\left(\mathrm{C}-1^{\prime}\right), 129.95\left(\mathrm{C}-2^{\prime}, \mathrm{C}-6^{\prime}\right), 133.32\left(\mathrm{C}-4^{\prime \prime}\right), 141.93\left(\mathrm{C}-5^{\prime \prime}\right)$, $153.45\left(\mathrm{C}-2^{\prime \prime}\right), 158.38\left(\mathrm{C}-4^{\prime}\right), 169.28$ (C-8), 170.12 (C7"), 170.93 (C-12), $174.01(\mathrm{C}-7), 184.58\left(\mathrm{C}-6^{\prime \prime}\right), 185.52\left(\mathrm{C}-3^{\prime \prime}\right)$. HSQC and HMBC correlations see Table 1. LC/MS $m / z 557.1$ ([M-H] $]^{-}$API-ES neg. mode).

6-[2-(2-Carbamoyl-3,6-dioxocyclohexa-1,4-dienylamino)-2-(4-hydroxyphenyl)-acetylaminol-penicillanic Acid 3b Synthesis and isolation as described above. Dark red solid. ${ }^{1} \mathrm{H}-\mathrm{NMR} \delta 1.43$ (s, 3H, H-9 or H-10), 1.52 (s, $3 \mathrm{H}, \mathrm{H}-9$ or H-10), 4.29 (s, 1H, H-3), 5.37 (d, J=4.1 Hz, 1H, H-5), 5.53 (dd, $J=4.1 \mathrm{~Hz}, 1 \mathrm{H}, \mathrm{H}-6), 5.93(\mathrm{~d}, J=6.8 \mathrm{~Hz}, 1 \mathrm{H}, \mathrm{H}-13), 6.56(\mathrm{~d}, J=10.3 \mathrm{~Hz}, 1 \mathrm{H}$, H-4"), 6.68 (d, $\left.J=10.3 \mathrm{~Hz}, 1 \mathrm{H}, \mathrm{H}-5^{\prime \prime}\right), 6.81$ (d, $J=8.5 \mathrm{~Hz}, 2 \mathrm{H}, \mathrm{H}-3^{\prime}, \mathrm{H}-5^{\prime}$ ), 7.18 (d, 1H, H-11), 7.28 (d, $\left.J=8.6 \mathrm{~Hz}, 2 \mathrm{H}, \mathrm{H}-2^{\prime}, \mathrm{H}-6^{\prime}\right), 9.07$ (s, 2H, H-8'), 13.11 (d, $1 \mathrm{H}, J=6.9 \mathrm{~Hz}, \mathrm{H}-14) .{ }^{13} \mathrm{C}-\mathrm{NMR}$ not measured due to the poor solubility or poor stability in deuterium solvents. LC/MS $m / z 513.0([\mathrm{M}-\mathrm{H}]$ API-ES neg. mode), $537.0\left([\mathrm{M}+\mathrm{Na}]^{+}, 553.0[\mathrm{M}+\mathrm{K}]^{+}\right.$API-ES pos. mode).

6-[2-(4-Hydroxyphenyl)-2-(2-methoxycarbonyl-3,6-dioxocyclohexa1,4-dienylamino)-acetylamino]-penicillanic Acid 3c Synthesis and isolation as described above. Dark red solid. ${ }^{1} \mathrm{H}-\mathrm{NMR} \delta 1.43(\mathrm{~s}, 3 \mathrm{H}, \mathrm{H}-9$ or H10), 1.50 (s, 3H, H-9 or H-10), 3.64 (br s, 3H, H- $8^{\prime \prime}$ ), 4.23 (s, 1H, H-3), 5.36 (d, $J=4.0 \mathrm{~Hz}, 1 \mathrm{H}, \mathrm{H}-5), 5.48$ (dd, $J=4.0 \mathrm{~Hz}, 1 \mathrm{H}, \mathrm{H}-6), 6.58$ (d, $J=10.1 \mathrm{~Hz}$, $\left.1 \mathrm{H}, \mathrm{H}-4^{\prime \prime}\right), 6.67$ (d, $\left.J=10.0 \mathrm{~Hz}, 1 \mathrm{H}, \mathrm{H}-5^{\prime \prime}\right), 6.78$ (d, $J=8.6 \mathrm{~Hz}, 2 \mathrm{H}, \mathrm{H}-3^{\prime}, \mathrm{H}-$ $\left.5^{\prime}\right), 7.19$ (d, $\left.J=8.6 \mathrm{~Hz}, 2 \mathrm{H}, \mathrm{H}-2^{\prime}, \mathrm{H}-6^{\prime}\right), 7.30$ (d, $\left.1 \mathrm{H}, \mathrm{H}-11\right),{ }^{13} \mathrm{C}-\mathrm{NMR}$ not measured due to the poor solubility or poor stability in deuterium solvents. LC/MS $m / z 528.1$ ([M-H] $]^{-}$API-ES neg. mode).

6-[2-(2-Ethoxycarbonyl-3,6-dioxocyclohexa-1,4-dienylamino)-2-(4-hydroxyphenyl)-acetylaminol-penicillanic Acid 3d Synthesis and isolation as described above. Dark red solid. ${ }^{1} \mathrm{H}-\mathrm{NMR} \delta 1.14$ (br, 3H, H-9"), 1.43 (s, 3H, H-9 or H-10), 1.51 (s, 3H, H-9 or H-10), 4.08 (br, 2H, H-8"), 4.29 (s, 1H, H-3), 5.36 (d, $J=4.1 \mathrm{~Hz}, 1 \mathrm{H}, \mathrm{H}-5), 5.51$ (dd, $J=4.1 \mathrm{~Hz}, 1 \mathrm{H}, \mathrm{H}-6$ ), 6.58 (d, $\left.J=10.3 \mathrm{~Hz}, 1 \mathrm{H}, \mathrm{H}-4^{\prime \prime}\right), 6.68$ (d, $\left.J=10.3 \mathrm{~Hz}, 1 \mathrm{H}, \mathrm{H}-5^{\prime \prime}\right), 6.78$ (d, $\left.J=8.6 \mathrm{~Hz}, 2 \mathrm{H}, \mathrm{H}-3^{\prime}, \mathrm{H}-5^{\prime}\right), 7.18$ (d, $\left.J=8.6 \mathrm{~Hz}, 2 \mathrm{H}, \mathrm{H}-2^{\prime}, \mathrm{H}-6^{\prime}\right), 7.34$ (d, $1 \mathrm{H}, \mathrm{H}-11),{ }^{13} \mathrm{C}-\mathrm{NMR}$ not measured due to the poor solubility or poor stability in deuterium solvents. LC/MS $m / z 542.1\left([\mathrm{M}-\mathrm{H}]^{-}\right.$API-ES neg. mode $), 566.0\left([\mathrm{M}+\mathrm{Na}]^{+}, 581.9[\mathrm{M}+\mathrm{K}]^{+}\right.$API-ES pos. mode $)$.

6-\{2-[2-(2-Hydroxyethylcarbamoyl)-3,6-dioxocyclohexa-1,4-dienylamino]-2-phenyl-acetylamino\}-penicillanic Acid 3e Synthesis and isolation as described above. Dark red solid. ${ }^{1} \mathrm{H}-\mathrm{NMR} \delta 1.42(\mathrm{~s}, 3 \mathrm{H}, \mathrm{H}-9$ or H$10), 1.51(\mathrm{~s}, 3 \mathrm{H}, \mathrm{H}-9$ or $\mathrm{H}-10), 3.39\left(\mathrm{~m}, J=5.5 \mathrm{~Hz}, 2 \mathrm{H}, \mathrm{H}-9^{\prime \prime}\right), 3.58$ (t, $\left.J=5.4 \mathrm{~Hz}, 2 \mathrm{H}, \mathrm{H}-10^{\prime \prime}\right), 4.29$ (s, $\left.1 \mathrm{H}, \mathrm{H}-3\right), 5.37$ (d, $\left.J=4.1 \mathrm{~Hz}, 1 \mathrm{H}, \mathrm{H}-5\right)$, 5.53 (dd, $J=4.1 \mathrm{~Hz}, 1 \mathrm{H}, \mathrm{H}-6), 6.03$ (d, $J=6.5 \mathrm{~Hz}, 1 \mathrm{H}, \mathrm{H}-13), 6.55$ (d, $J=$ $\left.10.1 \mathrm{~Hz}, 1 \mathrm{H}, \mathrm{H}-4^{\prime \prime}\right), 6.67$ (d, $\left.J=10.2 \mathrm{~Hz}, 1 \mathrm{H}, \mathrm{H}-5^{\prime \prime}\right), 7.39$ (m, 6H, H-2', H-3', H-4', H-5', H-6', H-11), 9.77 (t, 1H, H-8"), 13.28 (d, 1H, J=6.2 Hz, H-14). ${ }^{13} \mathrm{C}-\mathrm{NMR} \delta 27.04\left(\mathrm{C}-9\right.$ or C-10), $31.70\left(\mathrm{C}-9\right.$ or C-10), $42.10\left(\mathrm{C}-9^{\prime \prime}\right), 59.74$ (C-6), 61.47 (C-10"), 63.23 (C-13), 64.92 (C-2), 68.30 (C-5), 71.29 (C-3), $101.60\left(\mathrm{C}-1^{\prime \prime}\right), 128.49\left(\mathrm{C}-2^{\prime}, \mathrm{C}-6^{\prime}\right), 129.71\left(\mathrm{C}-4^{\prime}\right), 130.01\left(\mathrm{C}-3^{\prime}, \mathrm{C}-5^{\prime}\right)$, 133.39 (C-4"), 138.78 (C-1'), 141.87 (C-5"), 153.43 (C-2"), 169.83 (C-8), 170.11 (C7"), 170.80 (C-12), 173.90 (C-7), 184.74 (C-6"), 185.43 (C-3"). LC/MS $m / z 541.1\left([\mathrm{M}-\mathrm{H}]^{-}\right.$API-ES neg. mode), $565.0\left([\mathrm{M}+\mathrm{Na}]^{+}, 581.0\right.$ $[\mathrm{M}+\mathrm{K}]^{+}$API-ES pos. mode).

6-[2-(2-Carbamoyl-3,6-dioxocyclohexa-1,4-dienylamino)-2-phenylacetylamino]-penicillanic Acid 3f Synthesis and isolation as described above. Dark red solid. ${ }^{1} \mathrm{H}-\mathrm{NMR} \delta 1.42$ (s, 3H, H-9 or H-10), 1.52 (s, 3H, H9 or H-10), 4.31 (s, 1H, H-3), 5.37 (d, $J=4.1 \mathrm{~Hz}, 1 \mathrm{H}, \mathrm{H}-5), 5.54$ (dd, $J=$ $4.1 \mathrm{~Hz}, 1 \mathrm{H}, \mathrm{H}-6), 6.04$ (d, $J=6.9 \mathrm{~Hz}, 1 \mathrm{H}, \mathrm{H}-13), 6.57$ (d, $J=10.2 \mathrm{~Hz}, 1 \mathrm{H}, \mathrm{H}-$ $\left.4^{\prime \prime}\right), 6.68$ (d, $\left.J=10.2 \mathrm{~Hz}, 1 \mathrm{H}, \mathrm{H}-5^{\prime \prime}\right), 7.41$ (m, 6H, H-2', H-3', H-4', H-5', H6', H-11), 9.07 (s, 2H, H-8") 13.27 (d, 1H, J=6.2 Hz, H-14). LC/MS m/z $497.1\left([\mathrm{M}-\mathrm{H}]^{-}\right.$API-ES neg. mode $), 521.0\left([\mathrm{M}+\mathrm{Na}]^{+}, 536.9[\mathrm{M}+\mathrm{K}]^{+}\right.$ API-ES pos. mode).

6-[2-(2-Methoxycarbonyl-3,6-dioxocyclohexa-1,4-dienylamino)-2phenyl-acetylaminol-penicillanic Acid 3g Synthesis and isolation as described above. Dark red solid. ${ }^{1} \mathrm{H}-\mathrm{NMR} \delta 1.42$ (s, 3H, H-9 or H-10), 1.50 (s, $3 \mathrm{H}, \mathrm{H}-9$ or H-10), 3.62 (brs, $\left.3 \mathrm{H}, \mathrm{H}-8^{\prime \prime}\right), 4.28$ (s, 1H, H-3), 5.35 (d, $J=$ $4.0 \mathrm{~Hz}, 1 \mathrm{H}, \mathrm{H}-5), 5.50$ (dd, $J=4.0 \mathrm{~Hz}, 1 \mathrm{H}, \mathrm{H}-6), 6.58$ (d, $J=10.1 \mathrm{~Hz}, 1 \mathrm{H}, \mathrm{H}-$ $\left.4^{\prime \prime}\right), 6.67$ (d, J=10.2 Hz, 1H, H-5"'), 7.35 (m, 6H, H-2', H-3', H-4', H-5', H- 
6', H-11). LC/MS m/z 512.1 ([M-H] $]^{-}$API-ES neg. mode).

6-[2-(2-Ethoxycarbonyl-3,6-dioxocyclohexa-1,4-dienylamino)-2phenyl-acetylaminol-penicillanic Acid 3h Synthesis and isolation as described above. Dark red solid. ${ }^{1} \mathrm{H}-\mathrm{NMR} \delta 1.11$ (br, 3H, H-9"), 1.42 (s, 3H, H-9 or H-10), 1.53 (s, 3H, H-9 or H-10), 4.08 (br, 2H, H-8"), 4.31 (s, 1H, H3), 5.35 (d, $J=4.1 \mathrm{~Hz}, 1 \mathrm{H}, \mathrm{H}-5), 5.51$ (dd, $J=4.1 \mathrm{~Hz}, 1 \mathrm{H}, \mathrm{H}-6), 6.58$ (d, $\left.J=10.1 \mathrm{~Hz}, 1 \mathrm{H}, \mathrm{H}-4^{\prime \prime}\right), 6.68$ (d, $\left.J=10.1 \mathrm{~Hz}, 1 \mathrm{H}, \mathrm{H}-5^{\prime \prime}\right), 7.37$ (m, 6H, H-2', H3', H-4', H-5', H-6', H-11). LC/MS m/z 526.1 ([M-H $]^{-}$API-ES neg. mode), $550.0\left([\mathrm{M}+\mathrm{Na}]^{+}, 566.0[\mathrm{M}+\mathrm{K}]^{+}\right.$API-ES pos. mode $)$

Determination of Antibacterial Activity An agar diffusion method according to BURKHARDT ${ }^{24)}$ was used to determine antibacterial activity. Sterile Mueller-Hinton II-Agar in Stacker petri dishes (Becton Dickinson Microbiology systems, Cockeysville, U.S.A.) was inoculated with bacterial cells $\left(200 \mu \mathrm{l}\right.$ of bacterial cell suspension- $15 \times 10^{7}$ cells-on $20 \mathrm{ml}$ medium). The following bacterial strains were used: Bacillus megaterium SBUG 1152, Bacillus subtilis AWD 166, Staphylococcus aureus ATCC 6538, S. aureus Norddeutscher Epidemiestamm and Escherichia coli SBUG 1135. Besides these the agents were tested against the following multidrug resistant strains isolated from patients: $S$. aureus $315, S$. aureus $33490, S$. aureus 34289, S. aureus 36881, S. aureus 38418, S. aureus 39105, S. aureus $520, S$. aureus $526, S$. epidermidis $1068, S$. epidermidis $1071, S$. epidermidis 125, S. epidermidis 535, S. epidermidis 563, S. epidermidis 847, Enterococcus faecalis 769, E. faecalis 945, Pseudomonas aeruginosa 396, P. aeruginosa 595 and $P$. maltophilia 135 . The test samples were applied in different concentrations on sterile paper discs (Sensi-Disc, $6 \mathrm{~mm}$ diameter, Becton Dickinson Microbiology systems). Test concentrations were selected according to the concentration of the standard antibiotics (ampicillin, amoxicillin) on the Sensi discs. Plates were kept for $3 \mathrm{~h}$ in a refrigerator to enable prediffusion of the substances into the agar and were then incubated for $24 \mathrm{~h}$ at $37^{\circ} \mathrm{C}$. Average inhibition zone diameters were calculated from 3 replicates.

Cytotoxic Activity The cytotoxicity was determined by the neutral red uptake assay $^{25}$ ) using FL-cells, a human amniotic epithelial cell line. Only living cells are able to manage the active uptake of neutral red. FL-cells were cultivated in a 96 well microtiter plate $\left(10^{5}\right.$ cells $/ \mathrm{ml}$ Hepes modified Dulbecco, Sigma, $150 \mu \mathrm{l} /$ well) at $37^{\circ} \mathrm{C}$ in a humidified $5 \%$ carbon dioxide atmosphere. The Dulbecco's Modified Eagle's Medium (DMEM) was completed by L-glutamin (1\%, Sigma), penicillin G/streptomycin (1\%, Sigma) and FCS $(10 \%$, Biocrom). After $24 \mathrm{~h} 50 \mu \mathrm{l}$ of the test solution (test substance dissolved in $20 \mu \mathrm{l}$ DMSO under stirring in an ultrasonic bath for $5 \mathrm{~min}$ and then diluted with $1 \mathrm{ml}$ medium) or medium with equal amounts of DMSO (control) were added. After a further incubation for $72 \mathrm{~h}$ cells were washed three times with phosphate buffered saline solution (PBS). One hundred microliters neutral red solution (Sigma, 0.3\% in DMEM) was added per well. The cells were then incubated for $3 \mathrm{~h}$ at $37^{\circ} \mathrm{C}$, followed by another three times washing with PBS. One hundred microliters of a solution of acetic acid $(1 \%, \mathrm{v} / \mathrm{v})$ and ethanol $(50 \%, \mathrm{v} / \mathrm{v})$ in distilled water were added. After shaking for $15 \mathrm{~min}$ the optical density was measured at $492 \mathrm{~nm}$ with an Micro Screener LB 9260 (EG\&G Berthold, Bad Wildbad). The mean of three measurements for each concentration was determined $(n=3)$.

Animal Assays A "Staphylococcus-infected, immune suppressed mouse" model was established for the examination of in vivo effectiveness of in vitro selected drugs. In this model 8 weeks old female BALB/C mice (3 mice/group/assay) were pre-treated with cyclophosphamide $(250 \mathrm{mg} / \mathrm{kg}$ intra peritoneal (i.p.) day-3 and $100 \mathrm{mg} / \mathrm{kg}$ i.p. day-1, Sigma) to suppress the immune answer. Three days later they were infected with Staphylococcus aureus ATCC 6538 or Staphylococcus aureus 38418, i.p., in a lethal dose $\left(10^{10}-10^{12}\right.$ colony forming units (CFU)). The test agents were injected $30 \mathrm{~min}$ and $6 \mathrm{~h}$ after the infection with Staphylococcus aureus ATCC 6538 After the infection with Staphylococcus aureus 38418 the test agents were injected $30 \mathrm{~min}$ and $6 \mathrm{~h}$ after the infection, and the further injections are listed in Table 6 . The concentration of the test agents was selected according to therapeutic used doses of ampicillin. The antibiotic effectiveness was recognised within the next $2-6 \mathrm{~d}$.

Preliminary toxicity assays were done with one coupling product (3a) using 8 weeks old female BALB/C mice. The compound was injected at a dose of $2500 \mathrm{mg} / \mathrm{kg}$ i.p. Its influence was recognised by daily observation of the mice within the next $20 \mathrm{~d}$. In another set of experiments mice were treated with compound 3a $(50 \mathrm{mg} / \mathrm{kg}$ i.p. per injection) two times per day over a period of $14 \mathrm{~d}$

Stability against $\boldsymbol{\beta}$-Lactamase The stability of the coupling product 3a against two different $\beta$-lactamases was determined by comparison of its activity against Staphylococcus aureus SG10 in the agar diffusion test before and after treatment of the substances with $\beta$-lactamases. As control
$10 \mu \mathrm{g} / 50 \mu \mathrm{l}$ penicillin (10 I.E.) was pipetted into centered holes of the plates

One $\beta$-lactamase ( $\beta$-lactamase 1) has been produced by Escherichia coli DH5 (pBR322 in the presence of ampicillin and diluted 1:50 before use. The other $\beta$-lactamase ( $\beta$-lactamase 2$)$ has been produced by Bacillus subtilis AWD 166 and diluted 1:5 before use. Substances $(200 \mu \mathrm{g} / \mathrm{ml})$ were treated with $\beta$-lactamase for $30 \mathrm{~min}$ at $37^{\circ} \mathrm{C}$. Afterwards $50 \mu \mathrm{l}$ of the incubation mixture was tested for the remaining antibacterial activity as described above.

Determination of the Octanol-Water Partition Coefficient at $\mathrm{pH} 2$ $(\log \mathbf{D})$ The logarithm of the octanol-water partition ratio at $\mathrm{pH} 2(\log \mathrm{D})$ was determined according to Donovan and Pescatore ${ }^{26)}$ by using a HPLC system as described above with a column manufactured by Supelco (OPD50 -material, $20 \times 4.0 \mathrm{~mm}, 5 \mathrm{~mm}, 250 \AA$ pore size, Sigma). The column was operated between 18 and $24^{\circ} \mathrm{C}$ at a flow rate of $1 \mathrm{ml} / \mathrm{min}$. A solvent system consisting of methanol (eluent $\mathrm{A}$ ) and phosphoric acid, $0.1 \% \mathrm{pH} 2$ (eluent B), starting from an initial ratio of $10 \% \mathrm{~A}$ and $90 \% \mathrm{~B}$ and reaching $100 \% \mathrm{~A}$ within $9.4 \mathrm{~min}$, was used. A cocktail of internal standards was prepared by adding $440 \mathrm{mg}$ methylphenylsulfone to $380 \mu \mathrm{l}$ of toluene, followed by the addition of $150 \mathrm{ml}$ methanol. Approximately $0.5 \mathrm{mg}$ sample to be analysed was added to $0.5 \mathrm{ml}$ of this cocktail and $2 \mu \mathrm{l}$ was injected.

Acknowledgment We thank M. Kindermann, S. Siegert, B. Witt, and A. Neumann for providing NMR data, and S. Sokolowski for LC/MS data. Part of this work was supported financially by the Stiftung Industrieforschung (S574).

\section{References}

1) Shimizu M., Shiota S., Mizushima T., Ito H., Hatano T., Yoshida T. Tsuchiya T., Antimicrob. Agents Chemother., 45, 3198-3201 (2001).

2) Appelbaum P. C., Eur. J. Clin. Microbiol., 6, 367-377 (1987).

3) Critchley I. A., Karlowsky J. A., Draghi D. C., Jones M. E., Thornsberry C., Murfitt K., Sahm D. F., Antimicrob. Agents Chemother, 46, $550-555$ (2002).

4) Fluit A. C., Schmitz F. J., Jones M. E., Acar J., Gupta R., Verhoef J., Int. J. Infect. Dis., 3, 153-156 (1997).

5) Klugman K. P., Clin. Microbiol. Rev., 3, 171-196 (1990).

6) Fisher J. F., Meroueh S. O., Mobashery S., Chem. Rev., 105, 395-424 (2005).

7) Dalhoff A., Thomson C. J., Chemotherapy, 49, 105-120 (2003)

8) Waley S. G., "The Chemistry of $\beta$-Lactams," ed. by Page M. I., Blackie A. P., Chapman and Hall, London, United Kingdom, 1992, pp. $198-228$.

9) Helfand M. S., Bonomo R. A., Curr. Drug. Targets Infect. Disord., 3 , 9-23 (2003)

10) Helfand M. S., Bonomo R. A., Curr. Opin. Pharmacol., 5, 452-458 (2005).

11) Gianfreda L., Xu F., Bollag J. M., Biorem. J., 3, 1-25 (1999).

12) Mayer A. M., Staples C. A., Phytochemistry, 60, 551-565 (2002)

13) Xu F., "Encyclopedia of Bioprocess Technolog.: Ferm., Biocatalysis, and Bioseparation," ed. by Fleckinger M. C., Drew S. W., John Wiley \& Sons, New York, 1999, pp. 1545-1553.

14) Manda K., Hammer E., Mikolasch A., Niedermeyer T. H. J., Dec J., Jones A. D., Benesi A. J., Schauer F., Bollag J.-M., J. Mol. Catal. B: Enzym, 35, 86-92 (2005).

15) Mikolasch A., Hammer E., Jonas U., Popowski K., Stielow A., Schauer F., Tetrahedron, 58, 7589-7593 (2002).

16) Mikolasch A., Schauer F., J. Basic Microbiol., 43, 508-521 (2003).

17) Niedermeyer T. H. J., Mikolasch A., Lalk M., J. Org. Chem., 70 2002-2008 (2005).

18) Mothana R. A. A., Jansen R., Jülich W. D., Lindequist U., J. Nat Prod., 63, 416-418 (2000).

19) Fokialakis N., Magiatis P., Chinou I., Mitaku S., Tillequin F., Chem Pharm. Bull., 50, 413-414 (2002).

20) Yaoita Y., Danbara K., Kikuchi M., Chem. Pharm. Bull., 53, $1202-$ 1203 (2005).

21) Agematu H., Kominato K., Shibamoto N., Yoshioka T., Nishida H., Okamoto R., Shin T., Murao S., Biosci. Biotechnol. Biochem., 57, 1387-1388 (1993)

22) Agematu H., Tsuchida T., Kominato K., Shibamoto N., Yoshioka T., Nishida H., Okamoto R., Shin T., Murao S., J. Antibiot., 46, 141-148 (1993).

23) Anyanwutaku I., Petroski R., Rosazza J., Bioorg. Med. Chem., 2 , $543-551$ (1994) 
24) Burkhardt F., "Mikrobiologische Diagnostik," Georg Thieme Verlag, Stuttgart, New York, 1992

25) Lindl T., Bauer J., "Zell und Gewebekultur," Gustav Fischer Verlag, Jena, 1989.

26) Donovan S. T., Pescatore M. C., J. Chromatogr. A., 952, 47-61
(2002).

27) Tatsumi K., Freyer A., Minard R. D., Bollag J. M., Environ. Sci. Technol., 28, 210-215 (1994).

28) Tatsumi K., Freyer A., Minard R. D., Bollag J. M., Soil Biol. Biochem., 26, 735-742 (1994). 